\title{
Identificação e manejo de biótipos de Chloris polydactyla com resistência ou suscetibilidade diferencial ao glyphosate no Estado do Paraná1
}

\section{Identification and management of Chloris polydactyla biotypes with resistance or differential susceptibility to glyphosate in the state of Paraná}

Henrique Fabricio Placido ${ }^{2}$; Alfredo Junior Paiola Albrecht ${ }^{3}$; Rayssa Fernanda dos Santos ${ }^{2}$; Leandro Paiola Albrecht ${ }^{3}$; Andreos dos Santos Becker ${ }^{4}$; Arthur Arrobas Martins Barroso ${ }^{5}$; Ricardo Victória Filho ${ }^{6}$

Resumo - O surgimento de plantas daninhas resistentes tem aumentado os custos e prejudicado a produção dos cultivos pela interferência de plantas daninhas com o cultivo e perda de efetividade dos herbicidas de ampla utilização com amplo espectro de controle e baixo custo, como o glyphosate. Deste modo, o trabalho objetivou identificar populações de $C$. polydactyla resistente ou com suscetibilidade diferencial ao herbicida glyphosate, no Estado do Paraná, e estabelecer seu controle alternativo. Coletou-se biótipos com suspeita de resistência em municípios localizados nas regiões: oeste, centro-oeste e noroeste do estado. Instalou-se um ensaio de "screening" de populações, em delineamento inteiramente casualizado, com tratamentos de 0, 450, 650 e $900 \mathrm{~g}$ e.a. ha ${ }^{-1}$ do herbicida glyphosate, com 4 repetições, avaliando-se controle visual com 7, 14 e 21 dias após aplicação (DAA) e acúmulo de matéria fresca. Determinou-se o controle alternativo das diferentes populações com glyphosate; quizalofop; haloxyfop; tepraloxydim, clethodim e a associação destes herbicidas inibidores da ACCase com glyphosate, avaliando-se o controle visual aos 14, 21 e 28 DAA e redução da matéria fresca. O Paraná possui populações $C$. polydactyla que necessitam de altas doses de glyphosate para diminuir sua massa fresca ou atingir níveis adequados de controle, sendo um alerta para surgimento de populações resistentes. As populações estudadas desta espécie foram facilmente controladas com uso da associação glyphosate e clethodim, na dose comercial. É de suma importância rotacionar princípios ativos e mecanismos de ação na propriedade devido a características especificas da população alvo.

Palavras-chaves: capim branco; controle alternativo; graminicidas; resistência de plantas daninhas

Abstract - The emergence of resistant weeds has increased costs and affected the production of

\footnotetext{
${ }^{1}$ Recebido para publicação em 28/01/2016 e aceito em 26/07/2016.

${ }^{2}$ Escola Superior de Agronomia Luiz de Queiroz-USP, Av. Pádua Dias, 11- CEP 13418-900 - Piracicaba - SP, (placido.agronomia@gmail.com).

${ }^{3}$ Universidade Federal do Paraná - Setor Palotina; Professor do Departamento de Ciências Agronômicas - Rua Pioneiro, 2153, Jardim Dallas - CEP 85950-000 - Palotina (PR), Brasil, (ajpalbrecht@ yahoo.com.br).

${ }^{4}$ Universidade Federal do Paraná - Setor Palotina; Rua Pioneiro, 2153, Jardim Dallas - CEP 85950-000 - Palotina (PR), Brasil, (andreos.becker@gmail.com).

${ }^{5}$ Universidade Estadual Paulista Júlio de Mesquita Filho - UNESP - Campus Jaboticabal, Departamento de Biologia Aplicada a Agropecuária. Via de Acesso Prof. Paulo Donato Castellane s/n, CEP: 14884-900, Jaboticabal, SP. (arthuragro07@hotmail.com).

${ }^{6}$ Professor, Dr., titular do curso de agronomia na Escola Superior de Agronomia Luiz de Queiroz-USP, Av. Pádua Dias, 11- CEP 13418-900 - Piracicaba - SP, (placido.agronomia@gmail.com).
} 
crops for weed interference with cultivation and loss of effectiveness of herbicides widely used with large spectrums control and low cost, such as glyphosate. Thus, the study aimed to identify populations of $C$. polydactyla resistant or differential susceptibility to the herbicide glyphosate, at the State of Paraná, and establish its alternative control. It was collected biotypes suspected resistance in municipalities located in the West, Midwest and Northwest regions of state. Installed a test of resistant populations screening, in a completely randomized design with treatments 0,450 , 650 and $900 \mathrm{~g}$ a.e. ha ${ }^{-1}$ glyphosate herbicide, with 4 repetitions, assessing visual control with 7, 14 and 21 days after application - DAA and accumulation of fresh matter. Determined the alternative control by plants from different populations subjected to treatments: witness; glyphosate; quizalofop; haloxyfop; tepraloxydim, clethodim and the combination of these graminicides to glyphosate, by evaluating visual control at 14, 21 and 28 DAA and reduction of fresh matter. The state of Paraná has populations of $C$. polydactyla that require high doses of glyphosate to decrease its fresh weight or achieve adequate levels of control, and an alert to the emergence of resistant populations. The study populations of this species were easily controlled with the association of glyphosate and clethodim, in a commercial dose. It is extremely important to rotate active ingredients and mechanisms of action on the rural property due to specific characteristics of the target population.

Keywords: white grass; alternative control; graminicides; weed resistance

\section{Introdução}

A resistência de plantas daninhas a herbicidas é um processo evolutivo natural, em que o uso repetitivo do mesmo herbicida ou mecanismo de ação exerce forte pressão de seleção sobre uma população. A sobrevivência majoritária dos indivíduos resistentes, devido ao controle muito eficaz das plantas suscetíveis, dando origem a novas populações com altos níveis de resistência. A capacidade de sobreviver ao herbicida tem origem na variabilidade genética natural das plantas da espécie, sendo assim, o herbicida apenas seleciona os biótipos mais adaptados ao ambiente agrícola (Monquero, 2014).

O Brasil possui um total de 41 biótipos identificados como resistentes a herbicidas, representados por 28 espécies, onde 9 casos possuem resistência múltipla (Heap, 2016). Uma das mais recentes espécies identificadas com resistência ao glyphosate em nosso país é conhecida como capim-branco (Chloris polydactyla (L.) Sw.). Está relatada como resistente à molécula glyphosate desde o ano de 2013 (Barroso et al., 2013; Placido et al., 2013), porém sendo registrada e caracterizada no ano de 2014 (Brunharo, 2014; Heap, 2016).
Pertencente à família Poaceae, o gênero Chloris possui oito casos de resistência a herbicidas confirmados no mundo. Nos Estados Unidos foram identificadas duas populações da espécie C. Barbata (L.) Sw. resistente aos inibidores do Fotossistema II (C1/5; C2/7), na Austrália duas populações espécie $C$. truncata $\mathrm{R}$. Br. e três populações da espécie $C$. virgata Swartz foram considerada resistente grupo dos inibidores da enzima EPSPs (5-enolpiruvoilshikimato-3-fosfato sintetase) (G/9), e como anteriormente citado no Brasil um biótipo de $C$. polydactyla (L.) Sw. também resistente ao grupo de inibidores da enzima EPSPs (C/9) (Heap, 2016).

A critério de identificação e diferenciação da espécie $C$. polydactyla, em relação a espécie Chloris barbata também encontrada no Paraná temos as seguintes características $C$. polycatyla possui duas lemas aristadas, onde sua lema inferior é ciliada nas margens e carena, segunda lema glabra, terceira reduzida a escamas, não aristada, já a espécie $C$. Barbata possui três lemas aristadas, com sua lema inferior ciliada nas margens e carena, segunda e terceira lemas reduzidas a escamas glabras (Kissmann e Groth, 2007). 
O grupo de inibidores da enzima EPSPS tem como mecanismo de ação a inibição da síntese de aminoácidos aromáticos, fenilalanina, tirosina e triptofano, que são essenciais para formação de proteínas. Esta ação inibitória ocorre mais especificamente na enzima EPSPs que é decisiva na rota do ácido chiquímico, em que a mesma catalisa a reação shikimate-3phosphate (S3P) e phosphoenolpyruvate (PEP) formando 5-enolpyruvylshikimate-3-phosphate e phosphate (Geiger e Fuchs, 2002; Jaworski, 1972).

Esta espécie deve ser monitorada devido aos relatos da presença constante desta planta daninha nas de lavouras de soja, principalmente perto das estradas e áreas de reserva. Além disso, esta espécie já possui relato de resistência ao glyphosate, podendo a curto prazo se tornar um grande problema para o agricultor. Barroso et al. (2014-a) demonstraram que a espécie $C$. polydactyla mesmo sobre baixas densidades pode ter interferência significativa na soja e que a densidade de 20 plantas $\mathrm{m}^{-2}$ pode reduziu a biomassa seca da soja em até 44,1\%, dependendo da variedade utilizada.

Ademais ao identificar casos de resistência é essencial monitorar sua distribuição e propagação em áreas de cultivo de diferentes regiões, de modo que se tenha um monitoramento, estabelecendo-se modelos de risco de acordo com sua frequência (Burgos et al., 2013). Nesta temática ressalta-se a importância de novos estudos a partir da identificação da resistência, de modo que determinem a frequência de distribuição desta, além de estimar sua evolução.

Portanto, o objetivo deste trabalho foi identificar populações de $C$. polydactyla resistentes ou com suscetibilidade diferencial ao herbicida glyphosate, no Estado do Paraná, e também estabelecer seu controle alternativo.

\section{Material e Métodos}

As sementes dos biótipos da espécie $C$. polydactyla, foram coletadas em locais onde se relatou ineficiência no controle químico com o uso da molécula glyphosate. Através da indicação de produtores rurais, técnicos de cooperativas, além de localidades onde os biótipos não tiveram amplo contato com o herbicida, para estabelecer um comparativo.

Foram coletados um total de 23 biótipos nos municípios de Palotina (8), Assis Chateaubriand (3), Toledo (2), Maripá (1), Tupãssi (1), Francisco Alves (1), Iporã (1), Umuarama (1), Corbélia (1), Moreira Salles (2), Alto Piquiri (1) e Brasilândia do Sul (1), tal como mostrado na Figura 1.

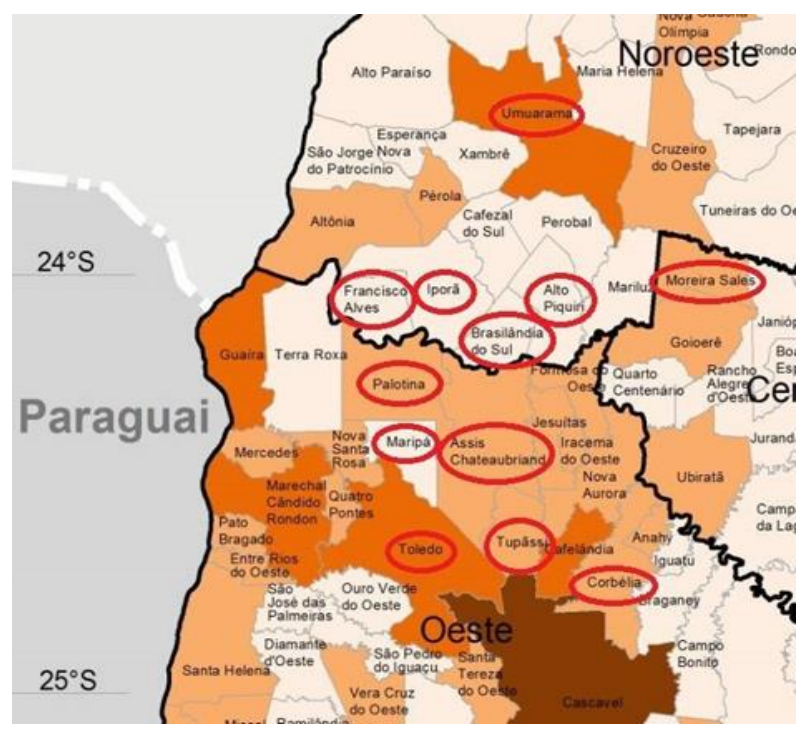

Figura 1. Localização dos municípios em que foram coletados os biótipos. Fonte: IPARDES, 2016.

As coletas ocorreram através da retirada manual das sementes de várias plantas selecionadas ao acaso dentro de uma população, colocando-as em sacos de papel kraft, anotandose local de coleta, as coordenas geográficas e cultura que estava estabelecida (Tabela 1). Em seguida, levou-se as mesmas para análise no Departamento de Produção Vegetal da Universidade Federal do Paraná.

\section{Experimento 1. Pré seleção ou screening das populações coletadas a campo}

Para pré-selecionar os biótipos com provável resistência, inicialmente semeou-se as populações em pequenas bandejas identificadas, 
preenchidas com substrato comercial crescimento inicial de próximo a $10 \mathrm{~cm}$, foram (Basaplant ${ }^{\circledR}$ ) e mantidas em casa de vegetação, transplantadas para vasos de $250 \mathrm{~mL}$, com temperatura média de $26^{\circ} \mathrm{C}$, umidade preenchidos com mistura de solo e substrato na relativa de $60 \%$ e irrigação diária programada de proporção de 1:1. $5 \mathrm{~mm} \mathrm{dia}{ }^{-1}$. Quando as plantas obtiveram um

Tabela 1. Relação dos biótipos e suas informações sobre as áreas de coleta.

\begin{tabular}{|c|c|c|c|c|}
\hline Biótipo & Município & Cultivo & Latitude & Longitude \\
\hline 1 & Toledo (2 irmãos) & Soja & $24^{\circ} 41^{\prime} 20,38^{\prime \prime} \mathrm{S}$ & $53^{\circ} 45^{\prime} 16,41^{\prime \prime} \mathrm{O}$ \\
\hline 2 & Palotina & Loteamento & $24^{\circ} 16^{\prime} 57,36^{*} \mathrm{~S}$ & $53^{\circ} 50^{\prime} 51,87^{\circ} \mathrm{O}$ \\
\hline 3 & Palotina & Loteamento & $24^{\circ} 17^{\prime} 18,83^{*} \mathrm{~S}$ & $53^{\circ} 50^{\prime} 20,96^{\circ} \mathrm{O}$ \\
\hline 4 & Iporã & Cana & $24^{\circ} 03^{\prime} 44^{\prime *} \mathrm{~S}$ & $53^{\circ} 44^{\prime} 43,92^{*} \mathrm{O}$ \\
\hline 5 & Maripá & Soja & $24^{\circ} 32^{\prime} 14,87^{*} \mathrm{~S}$ & $53^{\circ} 44^{\prime} 31,54^{\circ} \mathrm{O}$ \\
\hline 6 & Assis & Loteamento & $24^{\circ} 24^{\prime} 54,36^{*} \mathrm{~S}$ & $53^{\circ} 31^{\prime} 33,97^{\circ} \mathrm{O}$ \\
\hline 7 & Palotina & Soja & $24^{\circ} 15^{\prime} 28,78^{*} \mathrm{~S}$ & $53^{\circ} 51^{\prime} 52,30^{*} \mathrm{O}$ \\
\hline 8 & Toledo & Soja & $24^{\circ} 41^{\prime} 42,47^{*} \mathrm{~S}$ & $53^{\circ} 45^{\prime} 52,20^{\circ} \mathrm{O}$ \\
\hline 9 & Assis & Soja & $24^{\circ} 21^{\prime} 59,63^{*} \mathrm{~S}$ & $53^{\circ} 32^{\prime} 40,07^{\prime \prime} \mathrm{O}$ \\
\hline 10 & Assis - Brasilândia & Cana & $24^{\circ} 13^{\prime} 06,89^{*} \mathrm{~S}$ & $53^{\circ} 50^{\prime} 19,52^{*} \mathrm{O}$ \\
\hline 11 & Franscisco Alves & Soja & $24^{\circ} 03^{\prime} 44,71^{\prime *} \mathrm{~S}$ & $53^{\circ} 50^{\prime} 01,05^{\circ} \mathrm{O}$ \\
\hline 12 & Alto Piquiri & Cana & $24^{\circ} 31^{\prime} 58,54^{*} \mathrm{~S}$ & $53^{\circ} 44^{\prime} 57,90^{\circ} \mathrm{O}$ \\
\hline 13 & Tupãssi & Soja & $24^{\circ} 33^{\prime} 37^{\prime *} \mathrm{~S}$ & $53^{\circ} 48^{\prime} 49^{*} \mathrm{O}$ \\
\hline 14 & Umuarana & Pastagem & $23^{\circ} 54^{\prime} 11^{*} \mathrm{~S}$ & $53^{\circ} 04^{\prime} 46^{\circ} \mathrm{O}$ \\
\hline 15 & Morreira Salles & Soja & $23^{\circ} 54^{\prime} 58^{\prime \prime} \mathrm{S}$ & $53^{\circ} 05^{\prime} 33^{\prime \prime} \mathrm{O}$ \\
\hline 16 & Morreira Salles & Soja & $24^{\circ} 12^{\prime} \mathrm{S}$ & $53^{\circ} 32^{\prime} \mathrm{O}$ \\
\hline 17 & Assis & Soja & $24^{\circ} 18^{\prime} 1.92^{\prime \prime} \mathrm{S}$ & $53^{\circ} 46^{\prime} 51,34^{\prime \prime} \mathrm{O}$ \\
\hline 18 & Palotina & Soja & $24^{\circ} 16^{\prime} 14,87^{*} \mathrm{~S}$ & $53^{\circ} 52^{\prime} 48,09^{*} \mathrm{O}$ \\
\hline 19 & Palotina & Soja & $24^{\circ} 16^{\prime} 23,03^{*} \mathrm{~S}$ & $53^{\circ} 52^{\prime} 51,27^{\circ} \mathrm{O}$ \\
\hline 20 & Corbélia & Milho & $24^{\circ} 47^{\prime} 11,1^{\prime \prime S} \mathrm{~S}$ & $53^{\circ} 17^{\prime} 00.5^{\prime \prime} \mathrm{O}$ \\
\hline 21 & Palotina & Soja & $24^{\circ} 18^{\prime} 2,69^{\prime \prime} \mathrm{S}$ & $53^{\circ} 46^{\prime} 51,85^{\circ} \mathrm{O}$ \\
\hline 22 & Palotina (S. Camilo) & Soja & $24^{\circ} 13^{\prime} 14,1^{\prime \prime} \mathrm{S}$ & $53^{\circ} 53^{\prime} 1,56^{*} \mathrm{O}$ \\
\hline 23 & Palotina (S. Camilo) & Soja & $24^{\circ} 13^{\prime} 51,32^{\prime \prime} \mathrm{S}$ & $53^{\circ} 54^{\prime} 26,35^{\circ} \mathrm{O}$ \\
\hline
\end{tabular}

No momento que as plantas atingiram 4 folhas, antes do seu perfilhamento, aplicou-se para todas as populações as doses $0,450,650$, $900 \mathrm{~g} \mathrm{ha}^{-1}$ e.a. de glyphosate (Roundup Ready ${ }^{\circledR}$ $480 \mathrm{~g} \mathrm{~L}^{-1}$ e.a., SL, Monsanto), com 4 repetições, seguindo o delineamento inteiramente casualizado. Aplicou-se os tratamentos utilizando pulverizador costal, pressurizado com $\mathrm{CO}_{2}$, munido de barra com seis pontas do tipo leque, posicionada a $0,50 \mathrm{~m}$ do alvo, série 110.02, com pressão de 196,13 KPa proporcionando um volume de calda de $200 \mathrm{~L}$ $\mathrm{ha}^{-1}$.

A avaliação do controle obtido nos diferentes tratamentos foi realizada nos períodos 7, 14 e 21 dias após aplicação (DAA) seguindo a escala de avaliação visual de controle de plantas daninhas desenvolvida pela Associación Latino Americana de Malezas (Alam, 1974), que classifica a porcentagem de controle da seguinte forma, 0-40\% Controle "Pobre", 41$60 \%$ Controle "Regular", 61-70\% Controle "Suficiente", 71-80\% Controle "Bom", 81-90\% Controle "Muito Bom"; 91-100\% Controle "Excelente".

Após atendidas todas as pressuposições básicas para a análise de variância, aplicou-se o teste Scott-Knott $(P<0,05)$, método de comparação múltipla baseado em análise de agrupamento univariado (Silva, 2007). Com auxílio do programa Sisvar $^{\circledR}$ (Ferreira, 2011).

Para determinar o efeito das doses sobre o acúmulo de massa fresca, foi determinado a dose que reduziu a massa da matéria fresca em $50 \%\left(\mathrm{ED}_{50}\right)$. Os dados obtidos foram ajustados segundo o modelo de regressão não linear conforme Seefeldt et al. (1995), no programa SigmaPlot 10.0 (Wass, 2006), (Equação 1). 


$$
Y=c+\left\{\frac{d-c}{\left[1+(\mathrm{x} / \mathrm{g})^{b}\right]}\right\}
$$

Em que, " ${ }^{\prime}$ " representa a massa fresca ou seca percentual expressada com relação a testemunha, "c" e "d" são os coeficientes que representam os limites inferior e superior, respectivamente, " b" representa a inclinação da curva, "g" é a dose necessária para reduzir 50\% da massa da população e "x" é a dose de herbicida.

Ademais calculou-se o fator de resistência (FR) para o biótipo potencialmente resistente, pela Equação 2:

$$
F R=\frac{E D 50 \text { do biotipo Resistente }}{E D 50 \text { do biotipo Suscetível }}
$$

\section{Experimento 2. Utilização de herbicidas alternativos para controle de Chloris polydactyla}

Neste estudo utilizou-se biótipos considerados com controle intermediário, buscando estabelecer um controle preventivo, visto que nenhum dos biótipos testados obteve alto grau de resistência, foram utilizadas seis populações, as quais analisou-se a eficácia de herbicidas alternativos aplicados isolados ou associados com glyphosate. Quando as plantas atingiram 4 folhas totalmente expandidas aplicou-se os seguintes tratamentos isolados, glyphosate-sal isopropilamida (Roundup Ready $^{\circledR}, 480$ g L $^{-1}$ e.a., SL, Monsanto) na dose de $720 \mathrm{~g} \mathrm{ha}^{-1}$ e.a.; haloxyfop-p-methyl (Verdict ${ }^{\circledR}$ R, $120 \mathrm{~g} \mathrm{~L}^{-1}$ i.a., EC, Dow AgroSciences) na dose de $60 \mathrm{~g} \mathrm{ha}^{-1}$ i.a.; clethodim (Select 240 $\mathrm{EC}^{\circledR}, 240 \mathrm{~g} \mathrm{~L}^{-1}$ i.a., EC, Arysta LifeScience) na dose 108 g ha $^{-1}$ i.a.; tepraloxydim (Aramo ${ }^{\circledR}$ 200, $200 \mathrm{~g} \mathrm{~L}^{-1}$ i.a., EC, Basf) na dose $100 \mathrm{~g} \mathrm{ha}^{-1}$ i.a.; quizalofop-p-methyl (Targa $50 \mathrm{EC}^{\circledR}, 50 \mathrm{~g} \mathrm{~L}^{-1}$ i.a., EC, Basf) na dose $100 \mathrm{~g} \mathrm{ha}^{-1}$ i.a. Estes herbicidas supramencionado foram aplicados também em associação com o glyphosate, respeitando as mesmas dosagens dos produtos. Em todos os tratamentos acrescentou-se óleo mineral (Nimbus, $428 \mathrm{~g} \mathrm{~L}^{-1}$ i.a., EC, Syngenta) na dose de $0,5 \%$ do volume de calda, com 4 repetições por tratamento. A aplicação nos tratamentos deste experimento foi realizada do mesmo modo dos ensaios anteriores.

A classificação do controle obtido nos diferentes tratamentos foi realizada nos períodos 14, 21, 28 dias após aplicação (DAA) seguindo a escala de avaliação visual de controle de plantas daninhas desenvolvida pela Associación Latino Americana de Malezas (Alam, 1974). Completando-se os 28 DAA cortou-se a parte aérea das plantas para determinar o acúmulo da massa da matéria fresca em relação a testemunha.

Os dados foram submetidos a análise de variância $(P<0,05)$, e as médias comparadas pelo teste Scott-Knott $(P<0,05)$, método de comparação múltipla baseado em análise de agrupamento univariado, através do programa Sisvar $^{\circledR}$ (Ferreira, 2011).

\section{Resultados e Discussão}

\section{Experimento 1. Pré seleção ou screening das populações coletadas a campo}

$\mathrm{Na}$ avaliação de controle visual aos 7 DAA, notou-se que algumas populações obtiveram alta sensibilidade, pois, o controle destas populações foi classificado como "Muito Bom“" (Tabela 2).

Observa-se que aos 14 DAA até mesmo na menor dose houve um bom controle da maioria das populações, com médias entre bom e excelente, já para a maior dose aplicada, notase controle excelente para todas as populações, no entanto, para os biótipos B1, B4, B6, B11 e B22 as plantas não se encontravam completamente mortas, porém com alto grau de senescência. Os biótipos com menor controle foram B4 e B6, classificado como "Suficiente" (Tabela 2).

Aos 21 DAA considerado um período em que o herbicida glyphosate terminou de exercer efeitos sobre as plantas daninhas neste estádio, nota-se que vários biótipos (B9, B10 e B12 até B23) apresentaram alta suscetibilidade a molécula, em que mesmo com a dose de $450 \mathrm{~g}$ ha $^{-1}$ e.a. as populações em questão obtiveram 
controle total, já outros biótipos como B1, B2, porém com a dose de glyphosate de $650 \mathrm{~g} \mathrm{ha}^{-1}$ B7, B9 e B10 atingiram o mesmo controle, e.a.

Tabela 2. Controle visual aos 7 e 14 DAA de glyphosate para screening de biótipos de Chloris polydactyla com possível resistência coletados no Estado do Paraná, 2015.

\begin{tabular}{|c|c|c|c|c|c|c|c|c|}
\hline \multirow{3}{*}{ Biótipos } & \multicolumn{4}{|c|}{$\%$ de controle $-7 \mathrm{DAA}$} & \multicolumn{4}{|c|}{$\%$ de controle -14 DAA } \\
\hline & \multicolumn{4}{|c|}{ Dose g e.a. ha $^{-1}$} & \multicolumn{4}{|c|}{ Dose g e.a. ha ${ }^{-1}$} \\
\hline & 450 & 650 & 900 & Média** & 450 & 650 & 900 & Média** \\
\hline 1 & $40,00 \mathrm{f}$ & $47,50 \mathrm{e}$ & $48,75 \mathrm{~d}$ & $34,06 \mathrm{~g}$ & $86,25 \mathrm{c}$ & $91,00 \mathrm{c}$ & $96,75 \mathrm{~b}$ & $68,50 \mathrm{~b}$ \\
\hline 2 & $38,75 \mathrm{f}$ & $48,75 \mathrm{e}$ & $55,00 \mathrm{~d}$ & $35,63 \mathrm{~g}$ & $85,00 \mathrm{c}$ & $90,00 \mathrm{~d}$ & $100,00 \mathrm{a}$ & $68,75 \mathrm{~b}$ \\
\hline 3 & $48,75 \mathrm{~d}$ & $52,50 \mathrm{c}$ & $55,00 \mathrm{~d}$ & $39,06 \mathrm{f}$ & $86,75 \mathrm{c}$ & $95,75 \mathrm{~b}$ & $99,25 \mathrm{a}$ & $70,44 \mathrm{~b}$ \\
\hline 4 & $51,25 \mathrm{~d}$ & $50,00 \mathrm{c}$ & $52,50 \mathrm{~d}$ & $38,44 \mathrm{f}$ & $86,00 \mathrm{c}$ & $86,75 \mathrm{~d}$ & $92,50 \mathrm{~b}$ & $66,31 \mathrm{c}$ \\
\hline 5 & $45,00 \mathrm{e}$ & $47,50 \mathrm{~d}$ & $53,75 \mathrm{~d}$ & $36,56 \mathrm{~g}$ & $85,50 \mathrm{c}$ & $95,00 \mathrm{~b}$ & $98,50 \mathrm{a}$ & $68,75 \mathrm{~b}$ \\
\hline 6 & $51,25 \mathrm{~d}$ & $47,50 \mathrm{c}$ & $51,25 \mathrm{~d}$ & $37,50 \mathrm{f}$ & $86,00 \mathrm{c}$ & $88,00 \mathrm{~d}$ & $93,75 \mathrm{~b}$ & $69,75 \mathrm{c}$ \\
\hline 7 & $51,25 \mathrm{~d}$ & $67,50 \mathrm{c}$ & $78,75 \mathrm{~b}$ & $49,38 \mathrm{~d}$ & $86,00 \mathrm{c}$ & $94,50 \mathrm{~b}$ & $99,00 \mathrm{a}$ & $69,88 \mathrm{~b}$ \\
\hline 8 & $46,25 \mathrm{e}$ & $65,00 \mathrm{~d}$ & $78,75 \mathrm{~b}$ & $47,50 \mathrm{~d}$ & $82,50 \mathrm{c}$ & $93,25 \mathrm{c}$ & $99,00 \mathrm{a}$ & $68,69 \mathrm{~b}$ \\
\hline 9 & $50,00 \mathrm{~d}$ & $51,25 \mathrm{c}$ & $52,50 \mathrm{~d}$ & $38,44 \mathrm{f}$ & $95,25 \mathrm{~b}$ & $99,25 \mathrm{a}$ & $100,00 \mathrm{a}$ & $73,63 \mathrm{a}$ \\
\hline 10 & $43,75 \mathrm{e}$ & $50,00 \mathrm{~d}$ & $50,00 \mathrm{~d}$ & $35,94 \mathrm{~g}$ & $86,50 \mathrm{c}$ & $88,25 \mathrm{a}$ & $98,00 \mathrm{a}$ & $68,19 b$ \\
\hline 11 & $48,75 \mathrm{~d}$ & $65,00 \mathrm{c}$ & $65,00 \mathrm{c}$ & $44,69 \mathrm{e}$ & $87,50 \mathrm{c}$ & $95,75 \mathrm{~b}$ & $94,50 \mathrm{~b}$ & $69,44 \mathrm{~b}$ \\
\hline 12 & $55,00 \mathrm{~d}$ & $55,75 \mathrm{~d}$ & $66,50 \mathrm{c}$ & $44,31 \mathrm{e}$ & $90,00 \mathrm{c}$ & $89,25 \mathrm{~d}$ & $98,75 \mathrm{a}$ & $69,50 \mathrm{~b}$ \\
\hline 13 & $75,00 \mathrm{c}$ & $80,00 \mathrm{~b}$ & $85,00 \mathrm{a}$ & $60,00 \mathrm{c}$ & $100,00 \mathrm{a}$ & $100,00 \mathrm{a}$ & $100,00 \mathrm{a}$ & $75,00 \mathrm{a}$ \\
\hline 14 & $77,50 \mathrm{c}$ & $82,50 \mathrm{~b}$ & $85,00 \mathrm{a}$ & $61,25 \mathrm{c}$ & $100,00 \mathrm{a}$ & $100,00 \mathrm{a}$ & $100,00 \mathrm{a}$ & $75,00 \mathrm{a}$ \\
\hline 15 & $82,50 \mathrm{~b}$ & $78,75 \mathrm{~b}$ & $82,50 \mathrm{~b}$ & $60,94 \mathrm{c}$ & $100,00 \mathrm{a}$ & $100,00 \mathrm{a}$ & $100,00 \mathrm{a}$ & $75,00 \mathrm{a}$ \\
\hline 16 & $73,75 \mathrm{c}$ & $82,50 \mathrm{~b}$ & $83,75 \mathrm{a}$ & $60,00 \mathrm{c}$ & $95,00 \mathrm{~b}$ & $99,00 \mathrm{a}$ & $99,00 \mathrm{a}$ & $73,25 \mathrm{a}$ \\
\hline 17 & $83,75 \mathrm{~b}$ & $85,00 \mathrm{a}$ & $87,50 \mathrm{a}$ & $64,06 \mathrm{~b}$ & $99,50 \mathrm{a}$ & $100,00 \mathrm{a}$ & $100,00 \mathrm{a}$ & $74,88 \mathrm{a}$ \\
\hline 18 & $81,25 \mathrm{~b}$ & $82,50 \mathrm{~b}$ & $87,50 \mathrm{a}$ & $62,81 \mathrm{~b}$ & $99,25 \mathrm{a}$ & $98,50 \mathrm{a}$ & $100,00 \mathrm{a}$ & $74,44 \mathrm{a}$ \\
\hline 19 & $81,25 \mathrm{~b}$ & $86,25 \mathrm{a}$ & $85,00 \mathrm{a}$ & $63,13 b$ & $100,00 \mathrm{a}$ & $100,00 \mathrm{a}$ & $100,00 \mathrm{a}$ & $75,00 \mathrm{a}$ \\
\hline 20 & $83,75 \mathrm{~b}$ & $83,75 \mathrm{~b}$ & $85,00 \mathrm{a}$ & $63,13 b$ & $100,00 \mathrm{a}$ & $100,00 \mathrm{a}$ & $100,00 \mathrm{a}$ & $75,00 \mathrm{a}$ \\
\hline 21 & $83,75 \mathrm{~b}$ & $88,75 \mathrm{a}$ & $90,00 \mathrm{a}$ & $65,63 \mathrm{a}$ & $98,50 \mathrm{a}$ & $100,00 \mathrm{a}$ & $100,00 \mathrm{a}$ & $74,63 \mathrm{a}$ \\
\hline 22 & $87,50 \mathrm{a}$ & $87,50 \mathrm{a}$ & $87,50 \mathrm{a}$ & $65,63 \mathrm{a}$ & $100,00 \mathrm{a}$ & $99,00 \mathrm{a}$ & $95,00 \mathrm{~b}$ & $73,50 \mathrm{a}$ \\
\hline 23 & $87,50 \mathrm{a}$ & $88,75 \mathrm{a}$ & $88,75 \mathrm{a}$ & $66,25 \mathrm{a}$ & $100,00 \mathrm{a}$ & $100,00 \mathrm{a}$ & $100,00 \mathrm{a}$ & $75,00 \mathrm{a}$ \\
\hline $\mathrm{CV}(\%)$ & \multicolumn{4}{|c|}{6,54} & \multicolumn{4}{|c|}{3,32} \\
\hline
\end{tabular}

Suscetibilidade diferencial ou resistência cientifica ocorrem em uma espécie quando há diferença no controle de diferentes biótipos, porém se encontra abaixo da dose recomendada para controle desta espécie a campo (Barroso et al., 2014b). Estudos de Heap (2006) inferem que o surgimento de plantas resistentes é reflexo da evolução da suscetibilidade diferencial de populações de espécies de plantas daninhas.

Verificou-se que somente os biótipos B3, B4, B6, B8 e B11 não apresentaram 100\% de controle com a maior dose $\left(900 \mathrm{~g} \mathrm{ha}^{-1}\right.$ e.a. $)$, destacando-se os biótipos B4, B8 e B11 que estão entre as menores médias. Para esta dose notou-se certa variação entre a resposta das plantas ao herbicida dentro da mesma população, em que algumas repetições dos biótipos obtiveram um controle mais próximo ao suficiente, enquanto outras estavam completamente mortas com excelente controle, o que demostra alta variabilidade de resposta ao herbicida até mesmo na mesma população.

Para o parâmetro acúmulo de matéria fresca, houve menor redução na matéria fresca para os biótipos B4, B8 e B11 nas diferentes doses aplicadas, o biótipo 11 obteve em média $70 \%$ da matéria fresca da testemunha, portanto, este biótipo apresentou características de maior resistência ao herbicida glyphosate (Tabela 3).

Para este parâmetro os biótipos também apresentaram resposta diferencial entre as repetições na mesma população. Apesar da aparência clorótica e do alto grau de 
senescência, algumas plantas apresentaram acúmulo diferencial de matéria fresca, em que este pode estar relacionado com uma resposta fisiológica das plantas.

Tabela 3. Controle visual e acúmulo de matéria fresca aos 21 DAA de glyphosate para screening de biótipos de Chloris polydactyla com possível resistência coletados no Estado do Paraná, 2015.

\begin{tabular}{|c|c|c|c|c|c|c|c|c|}
\hline \multirow{3}{*}{ Biótipos } & \multicolumn{4}{|c|}{$\%$ de controle -21 DAA } & \multicolumn{4}{|c|}{ Matéria fresca (\%) } \\
\hline & \multicolumn{4}{|c|}{ Dose g e.a. ha ${ }^{-1}$} & \multicolumn{4}{|c|}{ Dose g e.a. ha ${ }^{-1}$} \\
\hline & 450 & 650 & 900 & Média** & 450 & 650 & 900 & Média** \\
\hline 1 & $86,50 \mathrm{~d}$ & $100,00 \mathrm{a}$ & $96,25 \mathrm{a}$ & $70,68 \mathrm{c}$ & $59,01 \mathrm{~b}$ & $26,48 \mathrm{~d}$ & $14,68 \mathrm{~b}$ & $50,04 \mathrm{c}$ \\
\hline 2 & $84,25 \mathrm{~d}$ & $100,00 \mathrm{a}$ & $100,00 \mathrm{a}$ & $71,06 \mathrm{c}$ & $47,97 \mathrm{~b}$ & $14,32 \mathrm{e}$ & $17,41 \mathrm{~b}$ & $44,93 \mathrm{c}$ \\
\hline 3 & $93,50 \mathrm{~b}$ & $97,50 \mathrm{a}$ & $99,25 \mathrm{a}$ & $72,56 \mathrm{~b}$ & $22,22 \mathrm{c}$ & $40,75 \mathrm{c}$ & $14,91 \mathrm{~b}$ & $44,47 \mathrm{c}$ \\
\hline 4 & $88,75 \mathrm{c}$ & $92,50 \mathrm{~b}$ & $100,00 \mathrm{a}$ & $70,31 \mathrm{c}$ & $52,98 \mathrm{~b}$ & $58,47 \mathrm{~b}$ & $33,41 \mathrm{a}$ & $61,22 \mathrm{~b}$ \\
\hline 5 & $88,75 \mathrm{c}$ & $100,00 \mathrm{a}$ & $100,00 \mathrm{a}$ & $72,18 \mathrm{~b}$ & $37,12 \mathrm{c}$ & $39,27 \mathrm{c}$ & $14,07 \mathrm{~b}$ & $47,62 \mathrm{c}$ \\
\hline 6 & $97,50 \mathrm{a}$ & $97,50 \mathrm{a}$ & $100,00 \mathrm{a}$ & $73,75 \mathrm{a}$ & $30,41 \mathrm{c}$ & $26,80 \mathrm{~d}$ & $30,42 \mathrm{a}$ & $46,91 \mathrm{c}$ \\
\hline 7 & $91,25 \mathrm{~b}$ & $100,00 \mathrm{a}$ & $100,00 \mathrm{a}$ & $72,81 \mathrm{~b}$ & $47,01 \mathrm{~b}$ & $28,48 \mathrm{~d}$ & $20,07 \mathrm{~b}$ & $48,89 \mathrm{c}$ \\
\hline 8 & $89,25 \mathrm{c}$ & $91,75 \mathrm{~b}$ & $97,25 \mathrm{a}$ & $69,56 \mathrm{c}$ & $75,81 \mathrm{a}$ & $53,30 \mathrm{~b}$ & $29,90 \mathrm{a}$ & $64,76 \mathrm{~b}$ \\
\hline 9 & $97,50 \mathrm{a}$ & $100,00 \mathrm{a}$ & $100,00 \mathrm{a}$ & $74,37 \mathrm{a}$ & $20,92 \mathrm{c}$ & $16,91 \mathrm{e}$ & $26,18 \mathrm{a}$ & $41,00 \mathrm{c}$ \\
\hline 10 & $97,50 \mathrm{a}$ & $100,00 \mathrm{a}$ & $100,00 \mathrm{a}$ & $74,37 \mathrm{a}$ & $29,12 \mathrm{c}$ & $13,51 \mathrm{e}$ & $7,92 \mathrm{~b}$ & $37,64 \mathrm{~d}$ \\
\hline 11 & $87,50 \mathrm{a}$ & $95,00 \mathrm{~b}$ & $98,50 \mathrm{a}$ & $70,25 \mathrm{a}$ & $90,36 \mathrm{a}$ & $72,45 \mathrm{a}$ & $38,59 \mathrm{a}$ & $75,35 \mathrm{a}$ \\
\hline 12 & $100,00 \mathrm{a}$ & $100,00 \mathrm{~b}$ & $100,00 \mathrm{a}$ & $75,00 \mathrm{a}$ & $15,38 \mathrm{~d}$ & $21,12 \mathrm{~d}$ & $11,78 \mathrm{~b}$ & $37,07 \mathrm{~d}$ \\
\hline 13 & $100,00 \mathrm{a}$ & $100,00 \mathrm{a}$ & $100,00 \mathrm{a}$ & $75,00 \mathrm{a}$ & $9,46 \mathrm{~d}$ & $7,51 \mathrm{e}$ & $11,14 \mathrm{~b}$ & $32,02 \mathrm{~d}$ \\
\hline 14 & $100,00 \mathrm{a}$ & $100,00 \mathrm{a}$ & $100,00 \mathrm{a}$ & $75,00 \mathrm{a}$ & $10,91 \mathrm{~d}$ & $8,23 \mathrm{e}$ & $10,94 \mathrm{~b}$ & $32,52 \mathrm{~d}$ \\
\hline 15 & $100,00 \mathrm{a}$ & $100,00 \mathrm{a}$ & $100,00 \mathrm{a}$ & $75,00 \mathrm{a}$ & $16,13 \mathrm{~d}$ & $28,84 \mathrm{~d}$ & $20,31 \mathrm{~b}$ & $41,32 \mathrm{c}$ \\
\hline 16 & $100,00 \mathrm{a}$ & $100,00 \mathrm{a}$ & $100,00 \mathrm{a}$ & $75,00 \mathrm{a}$ & $23,83 \mathrm{c}$ & 8,23 e & $20,75 \mathrm{~b}$ & $39,01 \mathrm{~d}$ \\
\hline 17 & $100,00 \mathrm{a}$ & $100,00 \mathrm{a}$ & $100,00 \mathrm{a}$ & $75,00 \mathrm{a}$ & $17,24 \mathrm{~d}$ & $16,63 \mathrm{e}$ & $20,34 \mathrm{~b}$ & $38,56 \mathrm{~d}$ \\
\hline 18 & $100,00 \mathrm{a}$ & $100,00 \mathrm{a}$ & $100,00 \mathrm{a}$ & $75,00 \mathrm{a}$ & $17,71 \mathrm{~d}$ & $19,15 \mathrm{~d}$ & $10,71 \mathrm{~b}$ & $36,89 \mathrm{~d}$ \\
\hline 19 & $100,00 \mathrm{a}$ & $100,00 \mathrm{a}$ & $100,00 \mathrm{a}$ & $75,00 \mathrm{a}$ & $11,96 \mathrm{~d}$ & $10,68 \mathrm{e}$ & $12,01 \mathrm{~b}$ & $33,66 \mathrm{~d}$ \\
\hline 20 & $100,00 \mathrm{a}$ & $100,00 \mathrm{a}$ & $100,00 \mathrm{a}$ & $75,00 \mathrm{a}$ & $22,77 \mathrm{c}$ & $23,94 \mathrm{~d}$ & $22,63 \mathrm{~b}$ & $42,34 \mathrm{c}$ \\
\hline 21 & $100,00 \mathrm{a}$ & $100,00 \mathrm{a}$ & $100,00 \mathrm{a}$ & $75,00 \mathrm{a}$ & $16,38 \mathrm{~d}$ & $10,15 \mathrm{e}$ & $12,39 \mathrm{~b}$ & $34,73 \mathrm{~d}$ \\
\hline 22 & $100,00 \mathrm{a}$ & $100,00 \mathrm{a}$ & $100,00 \mathrm{a}$ & $75,00 \mathrm{a}$ & $8,39 \mathrm{~d}$ & $19,72 \mathrm{~d}$ & $29,69 \mathrm{a}$ & $39,45 \mathrm{~d}$ \\
\hline 23 & $100,00 \mathrm{a}$ & $100,00 \mathrm{a}$ & $100,00 \mathrm{a}$ & $75,00 \mathrm{a}$ & $8,39 \mathrm{~d}$ & $12,99 \mathrm{e}$ & $10,95 \mathrm{~b}$ & $33,14 \mathrm{~d}$ \\
\hline $\mathrm{CV}(\%)$ & \multicolumn{4}{|c|}{3,37} & \multicolumn{4}{|c|}{26,46} \\
\hline
\end{tabular}

Através da análise de regressão nota-se que os B11 e B8, com possível resistência obtiveram menor decréscimo na matéria fresca conforme o aumento da dose herbicida com respectivamente $\mathrm{ED}_{50}$ de 1.179 e $745 \mathrm{~g} \mathrm{ha}^{-1}$ e.a., em relação ao suscetível que obteve $\mathrm{ED}_{50}$ de 326 $\mathrm{g} \mathrm{ha}^{-1}$ e.a. (Figura 2).

Deste modo, B11 e B8 obtiveram fatores de resistência de respectivamente 3,61 e 2,28 em relação ao B10, em que B11 e B8 necessitam de respectivamente o triplo e o dobro da dose aplicada no biótipo suscetível para também reduzir sua massa fresca em $50 \%$. O valor de $\mathrm{ED}_{50}$ de B11 para esta análise foi superior ao biótipo da espécie de $C$. polydactyla registrado como resistente por Brunharo (2014), que obtiveram um ED 50 de $826 \mathrm{~g} \mathrm{ha}^{-1}$ e.a.
Porém se faz necessário realizar novos estudos e curvas dose-resposta com a população F1 deste possível biótipo resistente (visto que este é segregante) para melhor ajuste do modelo da regressão determinando o ponto de $\mathrm{ED}_{50}$, e sendo possível determinar valores que reduzem o acúmulo de matéria fresca em $80 \%\left(\mathrm{ED}_{80}\right)$ e $95 \%$ (ED 95 ), com maior confiabilidade (Burgos et al., 2013).

Estudos de Barroso et al. (2014a) também identificam suscetibilidade diferencial para a espécie $C$. polydactyla, obtendo um $\mathrm{ED}_{80}$ de $452 \mathrm{~g} \mathrm{ha}^{-1}$ e.a. para sua população com maior resistência e $114 \mathrm{~g} \mathrm{ha}^{-1}$ e.a. para sua população mais suscetível. Brunharo (2014) estabelece a dose de $705 \mathrm{~g} \mathrm{ha}^{-1}$ e.a. do herbicida glyphosate, em que se obteve controle de $80 \%$ da planta de 
capim-branco, tanto em avaliações visuais quanto de matéria seca.

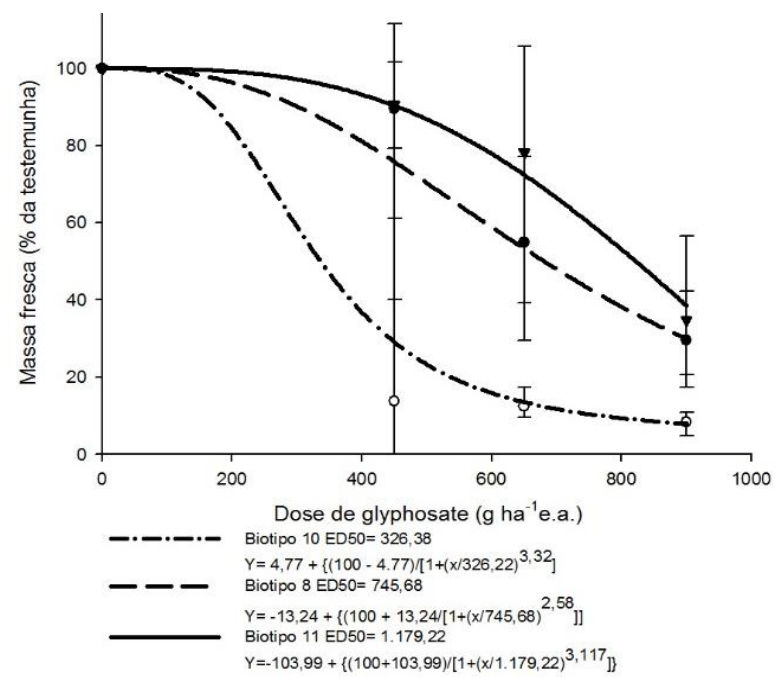

Figura 2. Acúmulo de massa fresca em relação a testemunha em biótipos de Chloris polydactyla (B10 suscetível vs B8 e B11 resistentes) submetidos a diferentes doses de glyphosate.

Para Placido et al. (2013) o estádio de desenvolvimento da planta é primordial para controle eficaz desta planta daninha, estes identificaram biótipos com suscetibilidade diferencial, porém notou-se que em aplicações com 4 folhas um biótipo obteve $\mathrm{ED}_{50}$ de 399,2 $\mathrm{g} \mathrm{ha}^{-1}$ e.a. e em aplicações com 4 perfilhos o mesmo biótipo aumento seu ED50 para 493,1 g ha ${ }^{-1}$ e.a.

Brunharo (2014) ao estudar um biótipo da espécie $C$. polydactyla resistente ao glyphosate, notou uma diminuição de $17 \%$ na absorção total do ${ }^{14} \mathrm{C}$-glyphosate no biótipo resistente em relação ao suscetível e analisando a translocação observou que a população resistente reteve na folha tratada $27 \%$ mais ${ }^{14} \mathrm{C}$ glyphosate as 12 horas após o tratamento (HAT) e $24 \%$ as 48 HAT, de modo que seu acúmulo de ácido chiquímico foi 2,54 vezes menor que a população sensível.

Brunharo et al. (2016) através de estudos de caracterização de mecanismo de resistência da espécie $C$. polydactyla, determinou que não há substituição de qualquer aminoácido na posição sequência, que codifica a enzima EPSPs, incluindo Thr102 e Pro106, além de não haver super expressão da enzima, em que o número de copias e de transcrição da mesma foi idêntico para os biótipos $\mathrm{S}$ e R.

\section{Experimento 2. Utilização de herbicidas alternativos para controle de Chloris polydactyla}

Para a primeira avaliação de controle visual aos 14 DAA, dentre os herbicidas aplicados de maneira isolada nos diferentes biótipos, o herbicida glyphosate obteve um bom controle inicial dos biótipos, tendo em vista que os mesmos são sensíveis a dose aplicada. O herbicida clethodim se destacou tendo controle superior para alguns biótipos (Tabela 4).

Aos 21 DAA os graminicidas associados ao glyphosate obtiveram um "excelente" controle sobre as diferentes populações estudadas, para os herbicidas aplicados de maneira isolada o herbicida haloxyfop obteve um controle "regular" para os biótipos 15 e 20, enquanto o herbicida tepraloxidim obteve controle "regular" para o biótipo 20 (Tabela 4).

No último período de avaliação com o uso do herbicida haloxyfop apenas as populações 15 e 20 obtiveram controle de $58,33 \%$ e $50,00 \%$ respectivamente, sendo os únicos biótipos com controle abaixo de "Suficiente". Neste período o herbicida quizalofop obteve maior dificuldade para controlar a população 15 com $66,67 \%$ de controle, sendo este "Suficiente" (Tabela 4).

Analisando o Acúmulo de massa de matéria fresca dentro do biótipo 14 nota-se que os tratamentos quizalofop e tetraploxydim obtiveram maior acúmulo de matéria fresca que os demais tratamentos de herbicidas, já o biótipo 22 obteve maior acúmulo de matéria fresca com o tratamento tetraploxydim, em relação aos demais tratamentos herbicidas (Tabela 5).

Para Brunharo (2014) avaliando controle visual aos 35 DAA para os mesmos herbicidas testados de maneira isolada, o mesmo obteve que dentre estes a molécula quizalofop teve o menor controle para as populações suscetíveis e 
resistentes com 30 e 46,25\% respectivamente, suscetível, já a população resistente não estes tratamentos também exerceram menor apresentou diferença entre a redução de matéria redução de matéria fresca na população fresca ocasionada por estes tratamentos.

Tabela 4. Controle visual aos 14, 21 e 28 DAA para herbicidas alternativos ou associados ao glyphosate aplicados em biótipos de Chloris polydactyla coletados no Estado do Paraná, 2015.

\begin{tabular}{|c|c|c|c|c|c|c|c|}
\hline \multirow{2}{*}{ Tratamentos } & \multicolumn{6}{|c|}{$\%$ de controle -14 DAA } & \multirow{2}{*}{ Média } \\
\hline & B13 & B14 & B15 & B16 & B20 & $\mathrm{B} 22$ & \\
\hline Testemunha & $0,00 \mathrm{Da}$ & $0,00 \mathrm{Ea}$ & $0,00 \mathrm{Ea}$ & $0,00 \mathrm{Da}$ & $0,00 \mathrm{Ca}$ & $0,00 \mathrm{Da}$ & $0,00 \mathrm{Fa}$ \\
\hline haloxyfop & $36,66 \mathrm{Cb}$ & $33,33 \mathrm{Db}$ & $28,33 \mathrm{Db}$ & $56,60 \mathrm{Ca}$ & $31,6 \mathrm{Bb}$ & $35,00 \mathrm{Cb}$ & $36,94 \mathrm{E}$ \\
\hline clethodim & $61,66 \mathrm{Ba}$ & $56,66 \mathrm{Ca}$ & $58,33 \mathrm{Ba}$ & $55,00 \mathrm{Ca}$ & $45,0 \mathrm{Ba}$ & $56,60 \mathrm{Ba}$ & $55,55 \mathrm{D}$ \\
\hline tepraloxydim & $51,66 \mathrm{Ba}$ & $45,00 \mathrm{Ca}$ & $45,00 \mathrm{Ca}$ & $50,00 \mathrm{Ca}$ & $35,0 \mathrm{Bb}$ & $33,33 \mathrm{Cb}$ & $43,33 \mathrm{E}$ \\
\hline quizalofop & $36,66 \mathrm{Cb}$ & $51,66 \mathrm{Ca}$ & $31,66 \mathrm{Db}$ & $58,33 \mathrm{Ca}$ & $33,33 \mathrm{Bb}$ & $36,60 \mathrm{Cb}$ & $41,38 \mathrm{E}$ \\
\hline glyphosate & $66,66 \mathrm{Bb}$ & $68,33 \mathrm{Bb}$ & $58,33 \mathrm{Bb}$ & $86,66 \mathrm{Ba}$ & $88,33 \mathrm{Aa}$ & $46,66 \mathrm{Bb}$ & $69,16 \mathrm{C}$ \\
\hline gly+haloxy & $60,00 \mathrm{Bc}$ & $81,66 \mathrm{Ab}$ & $71,66 \mathrm{Ab}$ & $96,66 \mathrm{Aa}$ & $78,33 \mathrm{Ab}$ & $78,33 \mathrm{Ab}$ & $77,77 \mathrm{~B}$ \\
\hline gly+cletho & 83,33 Аa & $90,00 \mathrm{Aa}$ & $76,66 \mathrm{Aa}$ & $96,66 \mathrm{Aa}$ & $91,66 \mathrm{Aa}$ & 93,33 Aa & $88,61 \mathrm{~A}$ \\
\hline gly+tepralo & $83,33 \mathrm{Aa}$ & $90,00 \mathrm{Aa}$ & $81,66 \mathrm{Aa}$ & $80,00 \mathrm{Ba}$ & $95,00 \mathrm{Aa}$ & $81,66 \mathrm{Aa}$ & $85,27 \mathrm{~A}$ \\
\hline gly+quizalo & 86,66 Aa & $85,00 \mathrm{Aa}$ & $73,33 \mathrm{Aa}$ & $81,66 \mathrm{Ba}$ & 83,33 Aa & 86,66 Aa & $82,27 \mathrm{~A}$ \\
\hline Média & $56,66 \mathrm{~b}$ & $60,16 \mathrm{~b}$ & $52,50 \mathrm{a}$ & $66,16 \mathrm{c}$ & $58,16 \mathrm{~b}$ & $54,83 \mathrm{a}$ & \\
\hline $\mathrm{CV}(\%)$ & & & & 15,66 & & & \\
\hline \multirow{2}{*}{ Tratamentos } & \multicolumn{6}{|c|}{$\%$ de controle $-21 \mathrm{DAA}$} & \multirow{2}{*}{ Média } \\
\hline & B13 & B14 & B15 & B16 & B20 & $\mathrm{B} 22$ & \\
\hline Testemunha & $0,00 \mathrm{Ca}$ & $0,00 \mathrm{Da}$ & $0,00 \mathrm{Fa}$ & $0,00 \mathrm{Ca}$ & $0,00 \mathrm{Ea}$ & $0,00 \mathrm{Ca}$ & $0,00 \mathrm{Fa}$ \\
\hline haloxyfop & $71,67 \mathrm{Bb}$ & $66,67 \mathrm{Cb}$ & $35,00 \mathrm{Ed}$ & $86,67 \mathrm{Ba}$ & $46,67 \mathrm{Dc}$ & $61,67 \mathrm{Bb}$ & $61,39 \mathrm{E}$ \\
\hline clethodim & 92,67 Aa & $78,33 \mathrm{Bb}$ & $88,33 \mathrm{Ba}$ & $91,67 \mathrm{Ba}$ & $86,66 \mathrm{Ba}$ & $88,33 \mathrm{Ba}$ & $87,67 \mathrm{C}$ \\
\hline tepraloxydim & $73,33 \mathrm{Ba}$ & $61,67 \mathrm{Cb}$ & $61,67 \mathrm{Db}$ & $81,67 \mathrm{Ba}$ & $43,33 \mathrm{Dc}$ & $66,67 \mathrm{Ab}$ & $64,72 \mathrm{E}$ \\
\hline quizalofop & $71,67 \mathrm{Bb}$ & $83,33 \mathrm{Ba}$ & $63,33 \mathrm{Db}$ & $85,00 \mathrm{Ba}$ & $66,67 \mathrm{Cb}$ & $91,67 \mathrm{Aa}$ & $76,94 \mathrm{D}$ \\
\hline glyphosate & $95,00 \mathrm{Aa}$ & $96,00 \mathrm{Aa}$ & $78,33 \mathrm{Cb}$ & $98,33 \mathrm{Aa}$ & $96,67 \mathrm{Aa}$ & $91,67 \mathrm{Aa}$ & $92,67 \mathrm{~B}$ \\
\hline gly+haloxy & $95,00 \mathrm{Aa}$ & $98,33 \mathrm{Aa}$ & 99,33 Aa & $100,00 \mathrm{Aa}$ & $91,67 \mathrm{Ba}$ & $100,00 \mathrm{Aa}$ & $97,39 \mathrm{~A}$ \\
\hline gly+cletho & $100,00 \mathrm{Aa}$ & $100,00 \mathrm{Aa}$ & $99,33 \mathrm{Aa}$ & $96,67 \mathrm{Aa}$ & $100,00 \mathrm{Aa}$ & $100,00 \mathrm{Aa}$ & $99,33 \mathrm{~A}$ \\
\hline gly+tepralo & $98,33 \mathrm{Aa}$ & $100,00 \mathrm{Aa}$ & 99,33 Аа & $90,00 \mathrm{Ba}$ & $100,00 \mathrm{Aa}$ & $97,67 \mathrm{Aa}$ & $97,56 \mathrm{~A}$ \\
\hline gly+quizalo & 99,33 Aa & $98,33 \mathrm{Aa}$ & $93,33 \mathrm{Ba}$ & $96,67 \mathrm{Aa}$ & $96,67 \mathrm{Aa}$ & $95,00 \mathrm{Aa}$ & $96,56 \mathrm{~A}$ \\
\hline Média & $79,70 \mathrm{~b}$ & $78,27 \mathrm{~b}$ & $71,80 \mathrm{~b}$ & $82,67 \mathrm{a}$ & $72,83 \mathrm{~b}$ & $79,27 \mathrm{~b}$ & \\
\hline $\mathrm{CV}(\%)$ & & & & 7,29 & & & \\
\hline \multirow{2}{*}{ Tratamentos } & \multicolumn{6}{|c|}{$\%$ de controle -28 DAA } & \multirow{2}{*}{ Média } \\
\hline & B13 & B14 & B15 & B16 & $\mathrm{B} 20$ & $\mathrm{~B} 22$ & \\
\hline Testemunha & $0,00 \mathrm{Ca}$ & $0,00 \mathrm{Ca}$ & $0,00 \mathrm{Ea}$ & $0,00 \mathrm{Ba}$ & $0,00 \mathrm{Da}$ & $0,00 \mathrm{Ba}$ & $0,00 \mathrm{Da}$ \\
\hline haloxyfop & $80,00 \mathrm{Bb}$ & $88,33 \mathrm{Bb}$ & $58,33 \mathrm{Dc}$ & $98,33 \mathrm{Aa}$ & $50,00 \mathrm{Cc}$ & 93,33 Aa & $78,05 \mathrm{C}$ \\
\hline clethodim & $95,00 \mathrm{Aa}$ & 98,33 Aa & $95,00 \mathrm{Aa}$ & $98,33 \mathrm{Aa}$ & $78,33 \mathrm{Bb}$ & $96,67 \mathrm{Aa}$ & $93,61 \mathrm{~B}$ \\
\hline tepraloxydim & $73,33 \mathrm{Ba}$ & $75,00 \mathrm{Ca}$ & $71,67 \mathrm{Ca}$ & $85,00 \mathrm{Ab}$ & $70,00 \mathrm{Ba}$ & $85,33 \mathrm{Ab}$ & $76,72 \mathrm{C}$ \\
\hline quizalofop & $95,00 \mathrm{Aa}$ & $96,67 \mathrm{Aa}$ & $66,67 \mathrm{Cb}$ & $97,67 \mathrm{Aa}$ & $90,00 \mathrm{Aa}$ & 97,67 Aa & $90,56 \mathrm{~B}$ \\
\hline glyphosate & 98,33 Aa & $100,00 \mathrm{Aa}$ & $83,33 \mathrm{Bb}$ & $100,00 \mathrm{Aa}$ & $100,00 \mathrm{Aa}$ & $97,67 \mathrm{Aa}$ & $96,56 \mathrm{~A}$ \\
\hline gly+haloxy & $88,33 \mathrm{Aa}$ & $100,00 \mathrm{Aa}$ & $100,00 \mathrm{Aa}$ & $100,00 \mathrm{Aa}$ & $96,67 \mathrm{Aa}$ & $100,00 \mathrm{Aa}$ & $97,50 \mathrm{~A}$ \\
\hline gly+cletho & $100,00 \mathrm{Aa}$ & $100,00 \mathrm{Aa}$ & 99,33 Аа & $98,33 \mathrm{Aa}$ & $100,00 \mathrm{Aa}$ & $100,00 \mathrm{Aa}$ & $99,61 \mathrm{~A}$ \\
\hline gly+tepralo & $100,00 \mathrm{Aa}$ & $100,00 \mathrm{Aa}$ & 99,33 Аа & $98,33 \mathrm{Aa}$ & $100,00 \mathrm{Aa}$ & $100,00 \mathrm{Aa}$ & $99,61 \mathrm{~A}$ \\
\hline gly+quizalo & $100,00 \mathrm{Aa}$ & $100,00 \mathrm{Aa}$ & $96,67 \mathrm{Aa}$ & 98,33 Аa & $100,00 \mathrm{Aa}$ & $96,67 \mathrm{Aa}$ & $98,61 \mathrm{~A}$ \\
\hline Média & $83,00 \mathrm{~b}$ & $85,83 \mathrm{a}$ & $77,03 \mathrm{c}$ & $87,43 \mathrm{a}$ & $78,50 \mathrm{c}$ & $86,70 \mathrm{a}$ & \\
\hline $\mathrm{CV}(\%)$ & & & & 7,97 & & & \\
\hline
\end{tabular}

Gly = glyphosate; haloxy $=$ haloxyfop; cletho = clethodim; tepralo = tepraloxydim; quizalo = quizalofop. *Médias seguidas de mesma letra maiúscula na coluna e minúscula na linha, não diferem entre si, pelo Teste de Scott-Knott $(P \leq 0,05)$.

A partir deste resultado de controle é de suma importância rotacionar princípios diferenciado para alguns biótipos, inferiu-se que ativos e mecanismos de ação na propriedade 
devido a características específicas da população alvo. Barroso (2014) constatou que a associação de glyphosate aos graminicidas quizalofop, haloxyfop e clethodim foram sinérgicas para o controle de Digitaria insularis resistente ao glyphosate, no entanto, este não avaliou o herbicida tepraloxydim. Em estudos sobre o controle da espécie $C$. polydactyla no cultivo de cana-de-açúcar, em que possui a maior infestação, Obara (2014), determinou que a associação dos herbicidas imazapic e imazapyr em pré-emergência foram eficientes no controle desta planta daninha, mantendo um período residual de 120 dias após a aplicação.

Tabela 5. Avaliação de matéria fresca (\% em relação à testemunha) aos 28 DAA para herbicidas alternativos ou associados ao glyphosate aplicados em biótipos de Chloris polydactyla coletados no Estado Paraná, 2015.

\begin{tabular}{|c|c|c|c|c|c|c|c|}
\hline \multirow{2}{*}{ Tratamentos } & \multicolumn{6}{|c|}{ Matéria fresca (\% em relação à testemunha) } & \multirow{2}{*}{ Média } \\
\hline & B13 & B14 & B15 & B16 & B20 & B22 & \\
\hline Testemunha & $100,00 \mathrm{Aa}$ & $100,00 \mathrm{Aa}$ & $100,00 \mathrm{Aa}$ & $100,00 \mathrm{Aa}$ & $100,00 \mathrm{Aa}$ & $100,00 \mathrm{Aa}$ & $100,00 \mathrm{Aa}$ \\
\hline haloxyfop & $43,46 \mathrm{Bb}$ & $34,15 \mathrm{Cb}$ & $36,96 \mathrm{Bb}$ & $15,42 \mathrm{Ba}$ & $42,29 \mathrm{Bb}$ & $19,35 \mathrm{Ca}$ & $31,94 \mathrm{~B}$ \\
\hline clethodim & $27,29 \mathrm{Ba}$ & $26,82 \mathrm{Ca}$ & $25,76 \mathrm{Ba}$ & $15,98 \mathrm{Ba}$ & $24,21 \mathrm{Ba}$ & $18,16 \mathrm{Ca}$ & $23,04 \mathrm{C}$ \\
\hline tepraloxydim & $46,90 \mathrm{Bb}$ & $55,62 \mathrm{Bb}$ & $28,65 \mathrm{Ba}$ & $20,78 \mathrm{Ba}$ & $34,82 \mathrm{Ba}$ & $45,35 \mathrm{Bb}$ & $38,69 \mathrm{~B}$ \\
\hline quizalofop & $44,89 \mathrm{Bb}$ & $40,82 \mathrm{Bb}$ & $47,32 \mathrm{Bb}$ & $20,15 \mathrm{Ba}$ & $31,95 \mathrm{Ba}$ & $20,42 \mathrm{Ca}$ & $34,26 \mathrm{~B}$ \\
\hline glyphosate & $30,93 \mathrm{Ba}$ & $22,89 \mathrm{Ca}$ & $31,39 \mathrm{Ba}$ & $15,22 \mathrm{Ba}$ & $17,59 \mathrm{Ba}$ & $16,97 \mathrm{Ca}$ & $22,50 \mathrm{C}$ \\
\hline gly+haloxy & $34,58 \mathrm{Ba}$ & $27,48 \mathrm{Ca}$ & $15,50 \mathrm{Ba}$ & $7,71 \mathrm{Ba}$ & $22,18 \mathrm{Ba}$ & $16,42 \mathrm{Ca}$ & $20,64 \mathrm{C}$ \\
\hline gly+cletho & $26,02 \mathrm{Ba}$ & $22,33 \mathrm{Ca}$ & $29,38 \mathrm{Ba}$ & $9,07 \mathrm{Ba}$ & $13,48 \mathrm{Ba}$ & $20,18 \mathrm{Ca}$ & $20,08 \mathrm{C}$ \\
\hline gly+tepralo & $25,01 \mathrm{Ba}$ & $16,70 \mathrm{Ca}$ & $24,45 \mathrm{Ba}$ & $14,00 \mathrm{Ba}$ & $17,10 \mathrm{Ba}$ & $20,13 \mathrm{Ca}$ & $19,23 \mathrm{C}$ \\
\hline gly+quizalo & $27,66 \mathrm{Ba}$ & $21,60 \mathrm{Ca}$ & $24,28 \mathrm{Ba}$ & $15,85 \mathrm{Ba}$ & $20,28 \mathrm{Ba}$ & $21,86 \mathrm{Ca}$ & $21,92 \mathrm{C}$ \\
\hline Média & $40,67 \mathrm{a}$ & $36,84 \mathrm{a}$ & $36,17 \mathrm{a}$ & $23,42 \mathrm{c}$ & $32,39 \mathrm{~b}$ & $29,88 \mathrm{~b}$ & \\
\hline $\mathrm{CV}(\%)$ & & & & 36,43 & & & \\
\hline
\end{tabular}

Gly = glyphosate; haloxy = haloxyfop; cletho = clethodim; tepralo = tepraloxydim; quizalo = quizalofop. ${ }^{*}$ Médias seguidas de mesma letra maiúscula na coluna e minúscula na linha, não diferem entre si, pelo Teste de Scott-Knott $(P \leq 0,05)$.

Através dos estudos realizados nota-se uma evolução no caso de resistência/suscetibilidade diferencial da espécie de $C$. polyctyla no Brasil onde indícios de novos casos estão surgindo cada vez mais, de modo que pesquisas que monitorem este crescimento são indispensáveis (Burgos et al., 2013). Outro fator interessante a ser ressaltado é que a mesma foi encontrada em diversas áreas adentrando a cabeceira das lavouras de soja, aveia e milho no estado do Paraná.

\section{Conclusões}

O Paraná possui populações de $C$. polydactyla que necessitam de altas doses de glyphosate para diminuir sua massa fresca ou atingir níveis adequados de controle, sendo um alerta para surgimento de populações resistentes. As populações estudadas desta espécie foram facilmente controladas com uso da associação glyphosate e clethodim, na dose comercial. É de suma importância rotacionar princípios ativos e mecanismos de ação na propriedade devido a características especificas da população alvo.

\section{Referências}

Asociación Latinoamericana de Malezas. Recomendaciones sobre unificación de los sistemas de evaluación en ensayos de control de malezas. ALAM, v.1, n.1, p.35-38, 1974.

Barroso, A.A.M. Caracterização genética e foliar de capim-amargoso resistente ao herbicida glyphosate e eficácia de seu controle com associação de herbicidas. 2014. 77 f. Dissertação (Mestrado em Fitotecnia) Escola Superior de Agricultura Luiz de Queiroz, USP, Piracicaba, 2014.

Barroso, A.A.M.; Albrecht, A.J.; Albrecht, L.P.; Villetti, H.L.; ORSO, G.; Cavalli, A.L.; Victoria-fillho, R. Competição entre a cultura da 
soja e a planta daninha Chloris polydactyla. Revista do Centro Universitário de Patos de Minas, v.5, p.82-90, 2014a.

Barroso, A.A.M.; Albrecht, A.J.P.; Reis, F.C.; Placido, H.F.; Krenchinski, F.H.; Victoria-filho, R. Efeito do biótipo e do estádio vegetativo no controle químico de capim-branco. In: Congreso latinoamericano de malezas, 21., 2013, Cancún, Mexico. Anais... Cancún: ASOMECIMA, 2013, p.500-505.

Barroso, A.A.M.; Albrecht, A.J.P.; Reis, F.C.; Placido, H.F.; Toledo, R.E.; Albrecht, L.P.; Victoria-filho, V. Different glyphosate susceptibility in chloris polydactyla accessions. Weed Technology, v.28, n.4, p.587-591, 2014b.

Brunharo, C.A.C.G. Resistência da planta daninha capim-branco (Chloris polydactyla) ao herbicida glyphosate. 2014. 151 f. Dissertação (Mestrado em Fitotecnia) - Escola Superior de Agricultura Luiz de Queiroz, USP, Piracicaba, 2014.

Brunharo, C.A.C.G.; Patterson, D.R.C.; Melo, M.S.C.; Nicolai, M.; Gaines, T.A.; Nissen, S.J.; Christoffoleti, P.J. Confirmation and mechanism of glyphosate resistance in tall windmill grass (Chloris elata) from Brazil. Pest Management Science, v.72, p.1758-1764, 2016.

Burgos, N.; Tranel, P.J.; STREIBIG, J.C.; DAVIS, V.M.; SHANER, D.; NORSWORTHY, J.K.; RITZ, C. Review: Confirmation of Resistance to Herbicides and Evaluation of Resistance Levels. Weed Science, v.61, n.1, p.4-20, 2013.

Ferreira, D.F. Sisvar: a computer statistical analysis system. Ciência e Agrotecnologia, v.35, n.6, p.1039-1042, 2011.

Geiger, D.R.; Fuchs, M.A. Inhibitors of aromatic amino acid biosynthesis (glyphosate). In: Böger, P.; Wakabayashi, K.; Hirai, K. (Ed.). Herbicide classes in development: mode of action, targets, genetic engineering, chemistry. Springer, Berlin - New York, 2002. v.21, cap.3, p.59-85.

Heap, I. (2006). Criteria for Confirmation of the Herbicide- Resistant Weeds. Diponível em: <www.weedscience.org/in.asp.>> Acesso: 22 Jul. 2016.

Heap, I. International Survey of Herbicide Resistant Weeds. Disponível em: <www.weedscience.org>> Acesso: 22 Jul. 2016.

Ipardes - Instituto Paranaense de Desenvolvimento Econômico e Social. Disponível em: <www.ipardes.gov.br/> Acesso: 22 Jul. 2016.

Jaworski, E.G. Mode of action of Nphosphonomethylglycine: Inhibition of aromatic amino acid biosynthesis. Journal of Agricultural and Food Chemistry, v.20, p.1195-1198, 1972.

Kissmann, K.G.; Groth, D. Plantas infestantes e nocivas. São Paulo: BASF, Tomo I, 2007, 606 p.

Monquero, P.A. Aspectos da biologia e manejo das plantas daninhas. São Carlos: RiMa Editora, 2014. 430 p.

Obara, F.E.B. Atributos do solo, presença de palha e estádio de desenvolvimento da canade-açúcar na aplicação, afetando a eficácia e seletividade dos herbicidas imazapic e imazapyr. 2014. 55 f. Dissertação (Mestrado em Fitotecnia) - Escola Superior de Agricultura "Luiz de Queiroz", USP, Piracicaba, 2014.

Placido, H.F.; González-Torralva, F.; Barroso, A.A.B.; Albrecht, A.J.P.; Menédez, J.; De Prado, R.A. Resistência a glifosato en biotipos de Chloris polydactyla (L.) SW. recolectados en Brasil. Revista Agropecuaria y Forestal APF, v.1, n.2, p.19-22, 2013.

Seefeldt, S.S., Jensen, J.E., Fuerst, P. Loglogistic analysis of herbicide dose response relationships. Weed Technology, v.9, n.2, p.218-227, 1995. 
Silva, C.M.R. Uso do teste de Scott-Knott e da análise de agrupamentos, na obtenção de grupos de locais para experimentos com cana-de-açúcar. 2007. 46 f. Dissertação (Mestrado em Estatística e Experimentação Agronômica) - Escola Superior de Agricultura Luiz de Queiroz, USP, Piracicaba, 2007.

Wass, J.A. Scientific Computing. v.23, ed.8, p.10, 2006. 